\title{
EPAS1 Gene Polymorphisms Are Associated With High Altitude Polycythemia in Tibetans at the Qinghai-Tibetan Plateau
}

\author{
Jin Xu, MD; Ying-Zhong Yang, PhD; Feng Tang, PhD; Qin Ga, MD; Wuren Tana, MD; Ri-Li Ge, MD, PhD \\ From the Research Center for High Altitude Medical Sciences, Qinghai University School of Medicine, Qinghai, China (Drs Xu, Yang, Tang, Ga, \\ Tana, and Ge); and the Department of Clinical Medicine, Qinghai University School of Medicine, Qinghai, China (Dr Xu).
}

\begin{abstract}
Objective.-To test the hypothesis that the polymorphisms in the EPAS1 gene are associated with the susceptibility to high altitude polycythemia (HAPC) in Tibetans at the Qinghai-Tibetan Plateau.

Methods.-We enrolled 63 Tibetan HAPC patients and 131 matched healthy Tibetans as a control group, from the Yushu area in Qinghai where the altitude is greater than $3500 \mathrm{~m}$. Eight singlenucleotide polymorphisms (SNPs) of the EPAS1 gene, including rs12619696, rs13420857, rs2881504, rs4953388, rs13419896, rs4953354, rs10187368, and rs7587138, were genotyped by the Sequenom MassARRAY SNP assay.

Results.-The frequencies of the G allele of EPAS1 SNP rs13419896 were significantly higher in the HAPC group than in the control group $(P<.05)$. Moreover, the A alleles of rs12619696 and rs4953354 were prevalent in the HAPC group, and their counterpart homozygotes were prevalent in the normal Tibetan group $(P<.05)$.

Conclusions.-Compared with normal Tibetans, Tibetans with HAPC are maladapted and have a different haplotype in EPAS1 SNPs rs12619696, rs13419896, and rs4953354.
\end{abstract}

Key words: EPAS1, polymorphism, high altitude polycythemia, Tibetans

\section{Introduction}

Increased numbers of circulating erythrocytes develop in high altitude dwellers to compensate for the hypoxia associated with high altitude. This phenomenon is termed high altitude polycythemia (HAPC), which is characterized by excessive erythrocytosis (males, hemoglobin $[\mathrm{Hb}] \geq 21 \mathrm{~g} / \mathrm{dL}$; females, $\mathrm{Hb} \geq 19 \mathrm{~g} / \mathrm{dL})$. HAPC is prevalent in $5 \%$ to $18 \%$ of the population on the Qinghai-Tibetan Plateau. ${ }^{1,2}$ HAPC leads to significant increase in blood viscosity, microcirculation disturbance, or even extensive organ damage. ${ }^{3,4}$ Although hypobaric hypoxia is likely to be a cause of HAPC at high altitude, the precise mechanisms underlying the pathogenesis of HAPC are not well understood.

The Tibetans on the Qinghai-Tibetan plateau live permanently at an altitude up to 3000 to $4500 \mathrm{~m}$; they possess heritable adaptations to the hypoxic

Corresponding author: Ri-Li Ge, MD, PhD, Department of Internal Medicine, Research Center for High Altitude Medical Sciences, Qinghai University School of Medicine, 16 Kunlun Road, Xining 810001, Qinghai, China (e-mail: geriligao@hotmail.com). environment, as indicated by lower hemoglobin levels, lower hematocrits, higher oxygen saturation of blood in infants, and high work performance. ${ }^{5-7}$ However, some Tibetans living at high altitude still show elevated hemoglobin concentration and even exhibit HAPC. ${ }^{8-11}$

Growing evidence suggests that the hypoxia-inducible factor (HIF) oxygen-signaling pathway plays an important role in the adaptation of Tibetans. ${ }^{12-17}$ The human EPAS1 gene is located on chromosome 2p21-p16 and encodes the oxygen-sensitive alpha subunit of HIF-2, which is a key regulator of chronic hypoxia by regulating a large number of genes involved in the cellular and systemic responses to hypoxia. These responses include erythropoiesis, iron homeostasis, pulmonary hypertension and remodeling, vascular permeability, and lung and placental development. ${ }^{18}$ Our recent study showed that the polymorphisms in the EPAS1 gene are associated with the susceptibility to high altitude pulmonary edema (HAPE) in the Han Chinese. ${ }^{19}$ However, the association of EPAS1 gene polymorphisms with HAPC in the Tibetan population remains unclear. 
Up to now, 8 EPAS1 single-nucleotide polymorphisms (SNPs), including rs12619696, rs13420857, rs2881504, rs4953388, rs13419896, rs4953354, rs10187368, and rs7587138, have been shown to be related to the adaptation to high altitude. ${ }^{13,20,21}$ To explore the potential role of EPAS1 polymorphisms in the pathogenesis of HAPC in Tibetans, we examined these 8 SNPs of the EPAS1 gene in 63 subjects with HAPC and 129 healthy controls, all Tibetans from the Yushu area in Qinghai province, where the altitude is greater than $3500 \mathrm{~m}$ above sea level.

\section{Methods}

\section{SUBJECTS}

A total of 63 patients with HAPC (mean age, $45.51 \pm$ 10.07 years) and 131 control subjects (mean age, 45.14 \pm 11.78 years) participated in this study. All participants lived in the Yushu area in southwest Qinghai province (altitude $3760 \mathrm{~m}$ ), and they were all Kangba Tibetans. All HAPC patients were diagnosed at Yushu People's Hospital between March 2011 and June 2013. The inclusion criteria were 1) an $\mathrm{Hb}$ concentration of at least $21 \mathrm{~g} / \mathrm{dL}$ for men and at least $19 \mathrm{~g} / \mathrm{dL}$ for women, and 2) that HAPC patients were local Tibetans normally living at 3600 to $4400 \mathrm{~m}$. Patients with other diseases having similar clinical manifestations were excluded. Healthy Tibetans matching the patients in age, sex, and working conditions were randomly selected from a physical examination at an outpatient clinic to serve as control subjects. None of the participants had a history of respiratory or cardiovascular disease, such as chronic obstructive pulmonary disease, pulmonary infection, asthma, shunt, valvular disease, congenital heart disease, or hypertensive heart disease. The research protocol was approved by the ethics committee at the Qinghai University School of Medicine (Xining, China). All participants in this study signed informed consent. Hemoglobin concentration and hematocrit (HCT) were determined from venous blood samples using the Mindray Hematology Analyzer (BC-2300; Mindray, Shenzhen, China), and oxygen saturation $\left(\mathrm{SaO}_{2}\right)$ levels were tested by pulse oximeter (Ohmeda 3700 Pulse Oximeter; Datex-Ohmeda, Boulder, CO, USA).

\section{DNA EXTRACTION AND GENOTYPING ASSAYS}

Genomic DNA was extracted from venous blood by Gentra Puregene Blood Kit (Qiagen, Hilden, Germany) according to standard procedures. All selected SNPs were genotyped by the Sequenom MassARRAY SNP assay (Capital Bio Corporation, Beijing, China). SNP loci-tested polymerase chain reaction (PCR) primers and single base extension primers were designed by using the Sequenom MassARRAY Assay Design Genotyping Software and Tools
(Sequenom, San Diego, CA, USA). The PCR reaction was performed under the following thermal cycling conditions: $94^{\circ} \mathrm{C}$ for 4 minutes, then $94^{\circ} \mathrm{C}$ for 20 seconds, $56^{\circ} \mathrm{C}$ for 30 seconds, and $72^{\circ} \mathrm{C}$ for 1 minute for 45 cycles, and $72^{\circ} \mathrm{C}$ for 4 minutes. PCR products were treated with shrimp alkaline phosphatase to remove free deoxyribonucleoside triphosphates, and single base extension reaction was performed, which consisted of $2.0 \mu \mathrm{L}$ of EXTEND MIX, $0.619 \mu \mathrm{L}$ of $\mathrm{dd}_{2} \mathrm{O}, 0.94 \mu \mathrm{L}$ of Extend primer mix, $0.2 \mu \mathrm{L}$ of iPLEX buffer plus, $0.2 \mu \mathrm{L}$ of iPLEX terminator, and $0.041 \mu \mathrm{L}$ of iPLEX enzyme (Sequenom, San Diego, CA, USA). The thermal cycling conditions were as follows: $94^{\circ} \mathrm{C}$ for 30 seconds, then $94^{\circ} \mathrm{C}$ for 5 seconds, $52^{\circ} \mathrm{C}$ for 5 seconds, and $80^{\circ} \mathrm{C}$ for 5 seconds for 40 cycles, and $72^{\circ} \mathrm{C}$ for 3 minutes. The MassARRAY Nanodispenser RS1000 (Capital Bio Corporation, Beijing, China) was used for dispensing the purified extension products onto a 384-element SpectroCHIP bioarray (Sequenom, San Diego, CA, USA), and mass spectrometric analysis was performed using the MALDI-TOF (matrix-assisted laser desorption/ionizationtime of flight) (Sequenom, San Diego, CA, USA). The results were analyzed using TYPER 4.0 software (Sequenom, San Diego, CA, USA).

\section{STATISTICAL ANALYSIS}

SPSS software (version 17.0; SPSS, Inc, Chicago, IL, USA) was used for statistical analysis. Allele frequencies were calculated based on genotype frequencies in HAPC and control groups, and the intergroup difference was estimated with the $\chi^{2}$ test. A probability value of less than .05 was considered significant. Deviations from the Hardy-Weinberg equilibrium (HWE) were assessed by processing the $\chi^{2}$ test for genotype frequency. Population genetic data were analyzed using Arlequin (version 2.000) software (http://cmpg.unibe.ch/software/arle quin35/). Genetic distances were used to measure the divergence between study groups. The genetic distance ranged between 0 and 1 , where 1 was complete divergence and 0 indicated no divergence. The smaller the genetic distance, the closer the genetic relationship. A variable used in association with genetic distance to express the proportion of genetic diversity attributable to genotype frequency differences among study groups is $\mathrm{F}_{\mathrm{ST}}{ }^{22}$ The odds ratio (OR), CIs, and probability values between the HAPC groups and control groups were calculated for alleles of polymorphic loci.

\section{Results}

\section{CHARACTERISTICS OF SUBJECTS}

The data for the sex, average age, $\mathrm{SaO}_{2}, \mathrm{Hb}$, and HCT for the HAPC and control groups are reported in Table 1. 
Table 1. Characteristics of the study groups

\begin{tabular}{lllllll}
\hline Study group & $N$ & Sex & Age (year) & SaO $_{2}(\%)$ & $H b(g / d L)$ & $H C T(\%)$ \\
\hline \multirow{2}{*}{ HAPC } & 35 & Male & $47.7 \pm 1.7$ & $83.4 \pm 0.9^{a}$ & $23.0 \pm 0.3^{a}$ & $66.6 \pm 2.1^{a}$ \\
& 28 & Female & $42.8 \pm 1.8$ & $85.1 \pm 0.9^{a}$ & $20.1 \pm 0.2^{a}$ & $59.9 \pm 0.8^{a}$ \\
Control & 74 & Male & $46.4 \pm 1.5$ & $88.8 \pm 0.4$ & $16.2 \pm 0.1$ & $46.6 \pm 0.6$ \\
& 55 & Female & $43.2 \pm 1.4$ & $87.3 \pm 0.6$ & $15.8 \pm 0.2$ & $41.6 \pm 2.1$ \\
\hline
\end{tabular}

The data represent the mean and SE of HAPC patients and healthy control subjects.

$\mathrm{HAPC}$, high altitude polycythemia; $\mathrm{SaO}_{2}$, arterial oxygen saturation; $\mathrm{Hb}$, hemoglobin; $\mathrm{HCT}$, hematocrit.

${ }^{a} P<.05$ vs control group.

The incidence of HAPC was much higher in men than in women, consistent with the preponderance of men with HAPC. The $\mathrm{SaO}_{2}$ was significantly lower, whereas $\mathrm{Hb}$ and HCT were significantly higher in the HAPC group compared with the control group $(P<.05)$.

\section{GENOTYPE AND ALLELE DISTRIBUTION}

The genotypic distributions and allelic frequencies of 8 EPAS1 SNPs (rs12619696, rs13420857, rs2881504, rs4953388, rs113419896, rs4953354, rs10187368, and rs7587138) in HAPC and control study groups are shown in Tables 2 and 3.

All these polymorphisms were found to be in HWE in both study groups (Table 3). The AA genotypes of rs12619696 and rs4953354 were significantly more prevalent among the HAPC group (8.2\% and $12.9 \%)$ than the control group $(2.3 \%$ and $3.9 \%)$ with an OR of 0.227 (95\% CI, 0.052 to $1.001 ; P=.035$ ) and 0.199 (95\% CI, 0.060 to $0.658 ; P=.004$ ), respectively. Furthermore, the genotype GG rs13419896 differed significantly between HAPC and control groups with an OR of 0.062 (95\% CI, 0.007 to $0.530 ; P=.001$; Table 2).

Furthermore, we found that there were significant differences in the allele frequency of the rs 12619696 SNP between the 2 groups $(P=.014$; Table 2$)$; the A allele was much more prevalent among the HAPC group (24.6\%) than the control group (14.3\%), with an OR of 0.513 (95\% CI, 0.299 to 0.881 ). The difference was significant when applying the $\mathrm{F}_{\mathrm{ST}}$ statistics on the genetic distance (.121; Table 3). The $\mathrm{G}$ allele for the rs13419896 SNP was significantly more prevalent among the HAPC group (27\%) than the control group $(14.7 \% ; P=.004$; Table 2), with an OR of $0.466(95 \%$ CI, 0.275 to 0.789 ), and the difference was significant when applying the $\mathrm{F}_{\mathrm{ST}}$ statistics on the genetic distance (.203; Table 3). We observed a significantly higher incidence of the A allele of the rs4953354 SNP in the HAPC group (34.7\%) than in the control group (19.0\%), with an OR of 0.442 (95\% CI, 0.272 to $0.716 ; P=.001$;
Table 2), and the difference was significant when applying the $\mathrm{F}_{\mathrm{ST}}$ statistics on the genetic distance (.410; Table 3). We also found a significant difference for the rs2881504 SNP (A/G) between the HAPC and control groups $(P<.001$; Table 2$)$.

We showed that the rs12619696 SNP was significantly associated with HAPC risk under the dominant model of inheritance (OR, $0.515 ; 95 \% \mathrm{CI}, 0.271$ to $0.981 ; P=.042)$. The rs 13419896 SNP was significantly associated with HAPC risk under the dominant model (OR, $0.506 ; 95 \%$ CI, 0.269 to $0.964 ; P=.034$ ) as well as the recessive model of inheritance (OR, 13.964; 95\% CI, 1.642 to $118.737 ; P=.002$; Table 2$)$. In addition, the rs4953354 SNP was significantly associated with HAPC risk both under the dominant (OR, 0.399; 95\% CI, 0.215 to $0.742 ; P=.003)$ and recessive models of inheritance (OR, 3.674; 95\% CI, 1.149 to $11.745 ; P=$ .020; Table 2).

\section{Discussion}

In a previous study we genotyped 207 SNPs of the EPAS1 gene in Chr2: 46304028-46851921 in a sample of 31 healthy Tibetans. The results suggested that the polymorphism in the EPAS1 gene is associated with adaptation to high altitude in Tibetans. ${ }^{15}$ Thus we hypothesized that Tibetans with HAPC may carry a different genotype and alleles, and we genotyped 8 SNPs of the EPAS1 gene, 7 of them located in the same region of Chr2: 46304028-46851921. We genotyped 8 SNPs of the EPAS1 gene by the SNP assay and analyzed the haplotypes in HAPC and control groups. Collectively, our results indicate that there are significant differences in 3 SNPs (rs12619696, rs13419896, and rs4953354) between the 2 groups.

A previous study reported that rs12619696 was associated with different patterns of adaptation to high altitude between Tibetans and Andeans. ${ }^{21}$ In this study, we found that both the AA genotype and A allele of rs12619696 were significantly more prevalent among the HAPC group (8.2\% and $24.6 \%)$ than the control group 
Table 2. Comparison of genotype distributions, allele frequencies, and association with HAPC risk in HAPC and control groups

\begin{tabular}{|c|c|c|c|c|c|}
\hline SNP & $\begin{array}{c}\text { Genotype or allele } \\
\text { associated with SNP }\end{array}$ & $H A P C(n \%)$ & Control $(n \%)$ & OR $(95 \% C I)$ & $P$ value \\
\hline \multicolumn{6}{|l|}{ rs12619696 } \\
\hline \multirow[t]{3}{*}{ Genotype } & GG & $36(59.0)$ & $95(73.6)$ & 1 & \\
\hline & $\mathrm{AG}$ & $20(32.8)$ & $31(24.0)$ & $0.587(0.297-1.160)$ & .123 \\
\hline & AA & $5(8.2)$ & $3(2.3)$ & $0.227(0.052-1.001)$ & $.035^{a}$ \\
\hline \multirow[t]{2}{*}{ Allele } & $\mathrm{G}$ & $92(75.4)$ & $221(85.7)$ & 1 & \\
\hline & A & $30(24.6)$ & $37(14.3)$ & $0.513(0.299-0.881)$ & $.014^{a}$ \\
\hline \multirow[t]{2}{*}{ Dominant model } & GG & $36(59.0)$ & $95(73.6)$ & 1 & \\
\hline & $\mathrm{AG}+\mathrm{AA}$ & $25(41.0)$ & $34(26.4)$ & $0.515(0.271-0.981)$ & $.042^{a}$ \\
\hline \multirow[t]{2}{*}{ Recessive model } & AA & $5(8.2)$ & $3(2.3)$ & 1 & \\
\hline & $\mathrm{AG}+\mathrm{GG}$ & $56(91.8)$ & $126(97.7)$ & $3.750(0.886-16.237)$ & .060 \\
\hline \multicolumn{6}{|l|}{ rs13420857 } \\
\hline \multirow[t]{3}{*}{ Genotype } & GG & $1(1.7)$ & $1(0.8)$ & 1 & \\
\hline & $\mathrm{CG}$ & $8(13.3)$ & $21(16.3)$ & $2.625(0.146-47.183)$ & .499 \\
\hline & $\mathrm{CC}$ & $51(85.0)$ & $107(82.9)$ & $2.098(0.129-34.220)$ & .595 \\
\hline \multirow[t]{2}{*}{ Allele } & G & $10(8.3)$ & $23(8.9)$ & 1 & \\
\hline & $\mathrm{C}$ & $110(91.7)$ & $235(91.1)$ & $0.929(0.427-2.019)$ & .852 \\
\hline \multirow[t]{2}{*}{ Dominant model } & GG & $1(1.7)$ & $1(0.8)$ & 1 & \\
\hline & $\mathrm{GC}+\mathrm{CC}$ & $59(98.3)$ & $128(99.2)$ & $2.169(0.133-35.283)$ & .577 \\
\hline \multirow[t]{2}{*}{ Recessive model } & $\mathrm{CC}$ & $51(85.0)$ & $107(82.9)$ & 1 & \\
\hline & $\mathrm{GC}+\mathrm{GG}$ & $9(15.0)$ & $22(17.1)$ & $1.165(0.501-2.710)$ & .723 \\
\hline \multicolumn{6}{|l|}{ rs2881504 } \\
\hline \multirow[t]{3}{*}{ Genotype } & GG & $3(4.8)$ & $7(5.4)$ & 1 & \\
\hline & $\mathrm{AG}$ & $18(28.6)$ & $44(34.1)$ & $1.048(0.243-4.509)$ & .950 \\
\hline & AA & $42(66.7)$ & $78(60.5)$ & $0.796(0.196-3.239)$ & .749 \\
\hline \multirow[t]{2}{*}{ Allele } & $\mathrm{G}$ & $84(19.0)$ & $58(22.5)$ & 1 & \\
\hline & A & $102(81)$ & $200(77.5)$ & $2.840(1.883-4.283)$ & $.000^{a}$ \\
\hline \multirow[t]{2}{*}{ Dominant model } & GG & $3(4.8)$ & $7(5.4)$ & 1 & \\
\hline & $\mathrm{AG}+\mathrm{AA}$ & $60(95.2)$ & $122(94.6)$ & $0.871(0.218-3.489)$ & .846 \\
\hline \multirow[t]{2}{*}{ Recessive model } & AA & $42(66.7)$ & $78(60.5)$ & 1 & \\
\hline & $\mathrm{AG}+\mathrm{GG}$ & $21(33.3)$ & $51(39.5)$ & $1.308(0.695-2.459)$ & .405 \\
\hline \multicolumn{6}{|l|}{ rs4953388 } \\
\hline \multirow[t]{3}{*}{ Genotype } & AA & $21(34.4)$ & $57(44.2)$ & 1 & \\
\hline & $\mathrm{AG}$ & $29(47.5)$ & $60(46.5)$ & $0.762(0.391-1.487)$ & .425 \\
\hline & GG & $11(18.0)$ & $12(9.3)$ & $0.402(0.154-1.049)$ & .058 \\
\hline \multirow[t]{2}{*}{ Allele } & A & $71(58.2)$ & $174(67.4)$ & 1 & \\
\hline & $\mathrm{G}$ & $51(41.8)$ & $84(32.6)$ & $0.672(0.431-1.048)$ & .079 \\
\hline \multirow[t]{2}{*}{ Dominant model } & $\mathrm{AA}$ & $21(34.4)$ & $57(44.2)$ & 1 & \\
\hline & $\mathrm{AG}+\mathrm{GG}$ & $40(65.6)$ & $72(55.8)$ & $0.663(0.352-1.248)$ & .202 \\
\hline Recessive model & GG & $11(18.0)$ & $12(9.3)$ & 1 & \\
\hline & $\mathrm{AG}+\mathrm{AA}$ & $50(92.0)$ & $117(90.7)$ & $2.145(0.887-5.185)$ & .085 \\
\hline rs13419696 & & & & & \\
\hline Genotype & AA & $34(55.7)$ & $92(71.3)$ & 1 & \\
\hline & $\mathrm{AG}$ & $21(34.4)$ & $36(27.9)$ & $0.634(0.325-1.234)$ & .178 \\
\hline & GG & $6(9.8)$ & $1(0.8)$ & $0.062(0.007-0.530)$ & $.001^{a}$ \\
\hline Allele & A & $89(73.0)$ & $220(85.3)$ & 1 & \\
\hline & $\mathrm{G}$ & $33(27.0)$ & $38(14.7)$ & $0.466(0.275-0.789)$ & $.004^{a}$ \\
\hline Dominant model & $\mathrm{AA}$ & $34(55.7)$ & $92(71.3)$ & 1 & \\
\hline & $\mathrm{AG}+\mathrm{GG}$ & $27(44.3)$ & $37(28.7)$ & $0.506(0.269-0.954)$ & $.034^{a}$ \\
\hline Recessive model & GG & $6(9.8)$ & $1(0.8)$ & 1 & \\
\hline & $\mathrm{AG}+\mathrm{AA}$ & $55(90.2)$ & $128(99.2)$ & $13.964(1.642-118.737)$ & $.002^{a}$ \\
\hline rs4953354 & & & & & \\
\hline Genotype & GG & $27(43.5)$ & $85(65.9)$ & 1 & \\
\hline & $\mathrm{AG}$ & $27(43.5)$ & $39(30.2)$ & $0.459(0.238-0.883)$ & $.019^{a}$ \\
\hline
\end{tabular}


Table 2 (continued)

\begin{tabular}{|c|c|c|c|c|c|}
\hline$\underline{S N P}$ & $\begin{array}{c}\text { Genotype or allele } \\
\text { associated with SNP }\end{array}$ & HAPC $(n \%)$ & Control ( $n \%)$ & OR $(95 \% C I)$ & $P$ value \\
\hline & AA & $8(12.9)$ & $5(3.9)$ & $0.199(0.060-0.658)$ & $.004^{a}$ \\
\hline \multirow[t]{2}{*}{ Allele } & G & $81(65.3)$ & $209(81.0)$ & 1 & \\
\hline & A & $43(34.7)$ & $49(19.0)$ & $0.442(0.272-0.716)$ & $.001^{a}$ \\
\hline \multirow[t]{2}{*}{ Dominant model } & GG & $27(43.5)$ & $85(65.9)$ & 1 & \\
\hline & $\mathrm{AG}+\mathrm{AA}$ & $35(56.5)$ & $44(34.1)$ & $0.399(0.215-0.742)$ & $.003^{a}$ \\
\hline \multirow[t]{2}{*}{ Recessive model } & AA & $8(12.9)$ & $5(3.9)$ & 1 & \\
\hline & $\mathrm{AG}+\mathrm{GG}$ & $35(87.1)$ & $124(96.1)$ & $3.674(1.149-11.745)$ & $.020^{a}$ \\
\hline \multicolumn{6}{|l|}{ rs10187368 } \\
\hline \multirow[t]{3}{*}{ Genotype } & GG & $55(91.7)$ & $124(96.1)$ & 1 & \\
\hline & AG & $5(8.3)$ & $5(3.9)$ & $0.444(0.123-1.595)$ & .203 \\
\hline & AA & $0(0.0)$ & $0(0.0)$ & & \\
\hline \multirow[t]{2}{*}{ Allele } & G & $115(95.8)$ & $253(98.1)$ & 1 & \\
\hline & A & $5(4.2)$ & $5(1.9)$ & $0.455(0.129-1.601)$ & .209 \\
\hline \multirow[t]{2}{*}{ Dominant model } & GG & $55(91.7)$ & $124(96.1)$ & 1 & \\
\hline & $\mathrm{AG}+\mathrm{AA}$ & $5(8.3)$ & $5(3.9)$ & $0.444(0.123-1.595)$ & .203 \\
\hline \multirow[t]{2}{*}{ Recessive model } & AA & $0(0.0)$ & $0(0.0)$ & 1 & \\
\hline & $\mathrm{AG}+\mathrm{GG}$ & $60(100)$ & $129(100)$ & & \\
\hline \multicolumn{6}{|l|}{ rs7587138 } \\
\hline \multirow[t]{3}{*}{ Genotype } & GG & $1(1.7)$ & $5(3.9)$ & 1 & \\
\hline & AG & 16 (26.7) & $31(24.0)$ & $0.388(0.042-3.604)$ & .391 \\
\hline & AA & $43(71.7)$ & $93(72.1)$ & $0.433(0.049-3.816)$ & .438 \\
\hline \multirow[t]{2}{*}{ Allele } & G & $18(15.0)$ & 41 (15.9) & 1 & \\
\hline & A & $102(85.0)$ & $217(84.1)$ & $0.934(0.512-1.705)$ & .824 \\
\hline \multirow[t]{2}{*}{ Dominant model } & GG & $1(1.7)$ & $5(3.9)$ & 1 & \\
\hline & $\mathrm{AG}+\mathrm{AA}$ & $59(98.3)$ & $124(96.1)$ & $0.420(0.048-3.679)$ & .420 \\
\hline \multirow[t]{2}{*}{ Recessive model } & AA & 43 (71.7) & $93(72.1)$ & 1 & \\
\hline & $\mathrm{AG}+\mathrm{GG}$ & $17(28.3)$ & $36(27.9)$ & $0.979(0.496-1.934)$ & .952 \\
\hline
\end{tabular}

HAPC, high altitude polycythemia; OR, odds ratio, SNP, single-nucleotide polymorphism.

${ }^{a} P<.05$ vs control.

(2.3\% and $14.3 \%$ ), with an OR of 0.227 (95\% CI, 0.052 to $1.001 ; P<.05)$ and 0.513 (95\% CI, 0.299 to 0.881 ; $P<.05)$. In addition, rs12619696 was significantly associated with HAPC risk under the dominant model of inheritance (OR, $0.515 ; 95 \% \mathrm{CI}, 0.271$ to 0.981 ; $P<.05)$.

For the SNP rs13419896, the A allele is proposed as being advantageous for Tibetans. ${ }^{14,22}$ In this study, the A allele was reported as $73 \%$ in Tibetans with HAPC and $85.3 \%$ in a control group who were Tibetans adapted to high altitude. The genotype AA differed significantly between the HAPC (55.7\%) and control groups (71.3\%). Therefore, the A allele of rs 13419896 is the allele that is advantageous for Tibetans to adapt to hypoxia at high altitude. A high incidence of the $\mathrm{G}$ allele is associated with HAPC in Tibetans. Indeed, the $G$ allele of rs13419896 was significantly more prevalent among the HAPC group (27\%) than the control group (14.7\%; $P<.05)$. The SNP rs13419896 was also significantly associated with HAPC risk under the dominant as well as the recessive model of inheritance.

For the SNP rs4953354, the G allele had a high incidence in healthy Tibetans. ${ }^{14,22}$ Interestingly, we observed a significantly lower incidence of the $G$ allele in the HAPC group $(65.3 \%)$ compared with the control group $(81.0 \%)$. However, the AA genotype was significantly more prevalent among the HAPC group (12.9\%) than the control group (3.9\%). The rs13419896 SNP was significantly associated with HAPC risk both under the dominant and recessive models of inheritance. Therefore, our results indicated that a high incidence of the A allele and AA genotype of rs4953354 is associated with the risk of HAPC in Tibetans.

In addition, we found significant differences between 2 groups as measured by $\mathrm{F}_{\mathrm{ST}}$ on the genetic distances for the 3 SNPs: rs12619696, rs13419896, and rs4953354. The divergences resulted from different genotypes of these SNPs in the 2 groups. 
Table 3. Comparison of Hardy-Weinberg Equilibrium (HWE) and genetic distance in HAPC and control groups

\begin{tabular}{llrlllll}
\hline & \multirow{2}{*}{$\begin{array}{l}\text { Study } \\
\text { SNoup }\end{array}$} & $N$ & HWE P value & SG & 1 & 2 \\
\cline { 5 - 7 } rs12619696 & HAPC & 61 & .365 & 1 & .511 & .500 \\
& Control & 129 & .802 & 2 & $.121^{a}$ & .247 \\
rs13420857 & HAPC & 60 & .324 & 1 & & \\
& Control & 129 & .802 & 2 & & \\
rs2881504 & HAPC & 63 & .559 & 1 & & \\
& Control & 129 & .808 & 2 & & \\
rs4953388 & HAPC & 61 & .858 & 1 & & \\
& Control & 129 & .502 & 2 & & \\
rs13419896 & HAPC & 61 & .319 & 1 & .488 & .573 \\
& Control & 129 & .207 & 2 & $.203^{a}$ & .252 \\
rs4953354 & HAPC & 62 & .760 & 1 & .284 & .707 \\
& Control & 129 & .843 & 2 & $.410^{a}$ & .309 \\
rs10187368 & HAPC & 60 & .736 & 1 & & \\
& Control & 129 & .822 & 2 & & \\
rs7587138 & HAPC & 60 & .723 & 1 & & \\
& Control & 129 & .251 & 2 & & \\
\hline
\end{tabular}

HAPC, high altitude polycythemia; SNP, single-nucleotide polymorphism; SG, study group; $\mathrm{F}_{\mathrm{ST}}$, proportion of genetic diversity attributable to genotype frequency differences among study groups.

${ }^{a} P<.05$ computed from $\mathrm{F}_{\mathrm{ST}}$ statistics.

However, we should note several limitations of this study. First, we did not measure other clinical parameters of the subjects, such as systolic blood pressure, diastolic blood pressure, mean arterial pressure, and pulmonary artery systolic pressure, which would provide more information on the significance of EPAS1 SNPs in Tibetans with HAPC. Second, we only focused on 8 SNPs of EPAS1 and did not screen other genes. Third, we examined a modest number of subjects in the study. More subjects will be included in the 2 groups to increase the power to detect the association of EPAS1 SNPs with HAPC in Tibetans.

\section{Acknowledgments}

This study was supported by grants from the National Basic Research Program of China (No. 2012CB518200), Program of International S\&T Cooperation of China (No. 2011DFA32720), Natural Science Foundation of China (No. 31160219), The High Altitude Medical Sciences Key Laboratory of Qinghai (2013-Z-Y-05), and The Key Laboratory Development Foundation of Qinghai (No. 2014-Z-Y-07 \& 2014-Z-Y-30).

\section{References}

1. Gallagher SA, Hackett PH. High-altitude illness. Emerg Med Clin North Am. 2004;22:329-355, viii.

2. Windsor JS, Rodway GW. Heights and haematology: the story of haemoglobin at altitude. Postgrad Med J. 2007;83:148-151.

3. Gao YQ. High Altitude Military Medicine. Chongqing, China: Chongqing Publishing Company; 2005.

4. León-Velarde F, Maggiorini M, Reeves JT, et al. Consensus statement on chronic and subacute high altitude diseases. High Alt Med Biol. 2005;6:147-157.

5. Wu T, Wang X, Wei C, et al. Hemoglobin levels in Qinghai-Tibet: different effects of gender for Tibetans vs. Han. J Appl Physiol. 2005;98:598-604.

6. Niermeyer S, Yang P, Shanmina, Drolkar, Zhuang J, Moore LG. Arterial oxygen saturation in Tibetan and Han infants born in Lhasa, Tibet. $N$ Engl $J$ Med. 1995;333:1248-1252.

7. Zhuang J, Droma T, Sutton JR, et al. Smaller alveolararterial $\mathrm{O}_{2}$ gradients in Tibetan than Han residents of Lhasa (3658 m). Respir Physiol. 1996;103:75-82.

8. Beall CM, Goldstein MC. Hemoglobin concentration of pastoral nomads permanently resident at 4,850-5,450 meters in Tibet. Am J Phys Anthropol. 1987;73:433-438.

9. Tang DJ, Ye YX, Li J. Analysis of clinical characteristics and JAK2V617F mutation of Tibetan people living at high altitudes with polycythemia [in Chinese]. Chin J Hematol. 2012;33:960-962.

10. Wu T. A Tibetan with chronic mountain sickness followed by high altitude pulmonary edema on reentry. High Alt Med Biol. 2004;5:190-194.

11. Wu TY. Chronic mountain sickness on the QinghaiTibetan plateau. Chin Med J (Engl). 2005;118:161-168.

12. Wang B, Zhang YB, Zhang F, et al. On the origin of Tibetans and their genetic basis in adapting high-altitude environments. PLOS ONE. 2011;6:e17002.

13. Beall CM, Cavalleri GL, Deng L, et al. Natural selection on EPAS1 (HIF2alpha) associated with low hemoglobin concentration in Tibetan highlanders. Proc Natl Acad Sci USA. 2010;107:11459-11464.

14. Peng Y, Yang Z, Zhang H, et al. Genetic variations in Tibetan populations and high-altitude adaptation at the Himalayas. Mol Biol Evol. 2011;28:1075-1081.

15. Simonson TS, Yang Y, Huff CD, et al. Genetic evidence for high-altitude adaptation in Tibet. Science. 2010;329:72-75.

16. Xu S, Li S, Yang Y, et al. A genome-wide search for signals of high-altitude adaptation in Tibetans. Mol Biol Evol. 2011;28:1003-1011.

17. Yi X, Liang Y, Huerta-Sanchez E, et al. Sequencing of 50 human exomes reveals adaptation to high altitude. Science. 2010;329:75-78.

18. van Patot MC, Gassmann M. Hypoxia: adapting to high altitude by mutating EPAS-1, the gene encoding HIF- $2 \alpha$. High Alt Med Biol. 2011;12:157-167. 
19. Yang YZ, Wang YP, Qi YJ, et al. Endothelial PAS domain protein $1 \mathrm{Chr} 2: 46441523(\mathrm{hg} 18)$ polymorphism is associated with susceptibility to high altitude pulmonary edema in Han Chinese. Wilderness Environ Med. 2013;24:315-320.

20. Bigham AW, Wilson MJ, Julian CG, et al. Andean and Tibetan patterns of adaptation to high altitude. Am J Hum Biol. 2013;25:190-197.
21. Hanaoka M, Droma Y, Basnyat B, et al. Genetic variants in EPAS1 contribute to adaptation to high-altitude hypoxia in Sherpas. PLoS ONE. 2012;7:e50566.

22. Holsinger KE, Weir BS. Genetics in geographically structured populations: defining, estimating and interpreting F(ST). Nat Rev Genet. 2009;10:639-650. 